\title{
The Megamaser Cosmology Project: precise black hole mass measurement and the implication for the $M_{\mathrm{BH}}-\sigma_{\star}$ relation
}

Cheng-Yu Kuo ${ }^{1}$, James A. Braatz ${ }^{2}$, James J. Condon ${ }^{2}$, Caterina M. V. Impellizzeri ${ }^{2}$, Kwok-Yung Lo ${ }^{2}$, Ingyin $\mathrm{Zaw}^{3}$, Christian Henkel $^{4}$, Mark J. Reid ${ }^{5}$, Jenny E. Greene ${ }^{6}$, Feng Gao ${ }^{7}$ and Wei Zhao ${ }^{7}$

${ }^{1}$ Academia Sinica Institute of Astronomy and Astrophysics, P.O. Box 23-141, Taipei 10617, Taiwan email: cykuo@asiaa.sinica.edu.tw

${ }^{2}$ National Radio Astronomy Observatory, 520 Edgemont Road, Charlottesville, VA 22903, USA

${ }^{3}$ New York University Abu Dhabi, Abu Dhabi, UAE

${ }^{4}$ Max-Planck-Institut für Radioastronomie, Auf dem Hügel 69, 53121 Bonn, Germany

${ }^{5}$ Harvard-Smithsonian Center for Astrophysics, 60 Garden Street, Cambridge, MA 02138, USA

${ }^{6}$ Department of Astrophysical Sciences, Princeton University, Princeton, NJ 08544, USA

${ }^{7}$ Shanghai Astronomical Observatory, 80 Nandan Road, Shanghai 200030, China

\begin{abstract}
We made dynamical black hole mass measurements from nineteen Seyfert 2 galaxies which host sub-parsec $\mathrm{H}_{2} \mathrm{O}$ maser disks using the $\mathrm{H}_{2} \mathrm{O}$ megamaser technique. The nearly perfect Keplerian rotation curves in many of these maser systems guarantee the high accuracy and precision of the black hole mass measurements. With the stellar velocity dispersion $\left(\sigma_{\star}\right)$ of the galaxy bulges measured with the Dupont $2.5 \mathrm{~m}$ telescope at Las Campanas Observatory in the South and the Apache Point Observatory (APO) 3.5m telescope in the North, we found that $\mathrm{H}_{2} \mathrm{O}$ maser galaxies, most of which host pseudo bulges rather than classical bulges, do not all follow the $\mathrm{M}_{B H}-\sigma_{\star}$ relation shown in the literature. This result is well consistent with the latest findings by Kormendy \& Ho (2013) that only early type galaxies and galaxies with classical bulges follow a tight $\mathrm{M}_{\mathrm{BH}}-\sigma_{\star}$ relation. Such a tight correlation may not exist in pseudo bulge galaxies.
\end{abstract}

Keywords. accretion disks, water maser, Seyfert galaxies, black hole mass

\section{Introduction}

The primary goal of the Megamaser Cosmology Project (MCP; Braatz et al. 2009; Reid et al. 2009a; Braatz et al. 2010) is to determine the Hubble constant $H_{0}$ to $\sim 3 \%$ accuracy in order to constrain the equation of state parameter $w$ of dark energy. In our endeavor to determine an accurate Hubble constant, we measure direct angular-diameter distances to galaxies in the Hubble flow with the $\mathrm{H}_{2} \mathrm{O}$ megamaser technique. This technique involves sub-milliarcsecond resolution imaging and acceleration measurements of $\mathrm{H}_{2} \mathrm{O}$ megamasers from nearly edge-on, sub-parsec gas disks at the centers of active galaxies. Since the maser disk is usually significantly smaller than the "gravitational sphere of influence" (Barth 2003) of the black hole (BH) at the center, the kinematics of the water masers provide a direct probe of the gravitational potential of the $\mathrm{BH}$, and the $\mathrm{BH}$ mass $\left(\mathrm{M}_{\mathrm{BH}}\right)$ can be measured with high precision from the rotation curve of the maser disk. Measuring $\mathrm{BH}$ masses is therefore a second important product of this project in addition to the distance determination. While we still need acceleration measurements for $\mathrm{H}_{2} \mathrm{O}$ masers to determine distances, the VLBI imaging alone is sufficient to measure accurate 
central BH masses, assuming distances to the maser galaxies. These accurate BH masses provide an important basis for testing the $\mathrm{M}_{B H}-\sigma_{\star}$ relation, a tight correlation between $\mathrm{BH}$ mass and the velocity dispersion of stars $\left(\sigma_{\star}\right)$ in the galaxy bulge (e.g. Ferrarese \& Merritt 2000; Gebhardt et al. 2000; Gütekin et al. 2009, Kormendy \& Ho 2013) and thought to be a manifestation of a causal connection between the formation and evolution of the black hole and the bulge. Since $\mathrm{H}_{2} \mathrm{O}$ megamasers are usually found in late type galaxies and $\mathrm{BH}$ masses measured from $\mathrm{H}_{2} \mathrm{O}$ usually range from $\sim 10^{6}-10^{7.5} \mathrm{M}_{\odot}$, these $\mathrm{BH}$ masses play an important role to test whether the $\mathrm{M}_{B H}-\sigma_{\star}$ relation defined by early type galaxies also holds for late type galaxies.

\section{The sample and observations}

We searched for circumnuclear $\mathrm{H}_{2} \mathrm{O}$ maser disks from a sample of Seyfert 2 galaxies drawn from the SDSS survey (Data Release 7), the 6dF survey, and the 2MRS survey. Since the start of the MCP in 2006, we have observed more than 3000 Seyfert galaxies with recession velocities below $15000 \mathrm{~km} \mathrm{~s}^{-1}$. Water masers have been detected in 162 galaxies (see https://safe.nrao.edu/wiki/bin/view/Main/MegamaserCosmologyProject). Most of the megamasers originate in active galactic nuclei (Lo 2005) and about 30 galaxies show spectra suggestive of emission from sub-parsec scale, edge-on, circumnuclear disks. In the MCP, we conducted VLBI observations of nineteen megamaser disks with the Very Long Baseline Array augmented by the 100-m Green Bank Telescope and the Effelsberg 100-m telescope. We have obtained their images with sub-milliarcsecond resolution and use their rotation curves to measure BH masses.

The VLBI plays a crucial role in measuring BH masses with high precision especially in galaxies with lighter supermassive black holes (i.e. $M_{\bullet} \sim 10^{6}-10^{7} M_{\odot}$ ). The critical advantage provided by VLBI is angular resolution two orders of magnitude higher than the best optical resolution. For any given galaxy with a nearly constant central mass density of stars, $M_{\mathrm{BH}}$ is proportional to $R_{\mathrm{inf}}^{3}$, where $R_{\text {inf }}$ is the radius of the gravitational sphere of influence of $\mathrm{BH}$ (Barth et al. 2003). So, a factor of 100 increase in resolution permits measurements of masses up to $10^{6}$ times smaller. Similarly, the central density limits that can be set are up to $10^{6}$ times higher, high enough to rule out extremely dense star clusters as the massive objects at the center of many megamaser disks presented here based on dynamical argument (see Kuo et al. 2011).

\section{BH masses and their implication for the $M-\sigma$ relation}

Among the 19 maser galaxies for which we took VLBI data and have finished data analysis for BH mass measurements, we have published the VLBI maps (see Figure 1) of the water maser spots in seven systems (i.e. UGC 3789, NGC 1194, NGC 2273, NGC 2960, NGC 4388, NGC 6264, and NGC 6323; see also Kuo et al. 2011). Publications of VLBI maps for the other 12 maser galaxies are in preparation (Gao et al. in prep.; Zhao et al. in prep.). The typical size of the observed maser disks is typically less than $1 \mathrm{pc}$ as expected and all well-defined maser disks show warped structure. To obtain the BH mass, we first fit a Keplerian rotation curve to each observed maser disk:

$$
\left|v_{\mathrm{K}}\right|=v_{1}\left(\frac{\theta}{1 \mathrm{mas}}\right)^{-1 / 2},
$$

where $\left|v_{\mathrm{K}}\right|$ is the orbital velocity (after relativistic corrections) of the high velocity masers and $v_{1}$ is the orbital velocity at a radius 1 mas from the dynamical center. With the 

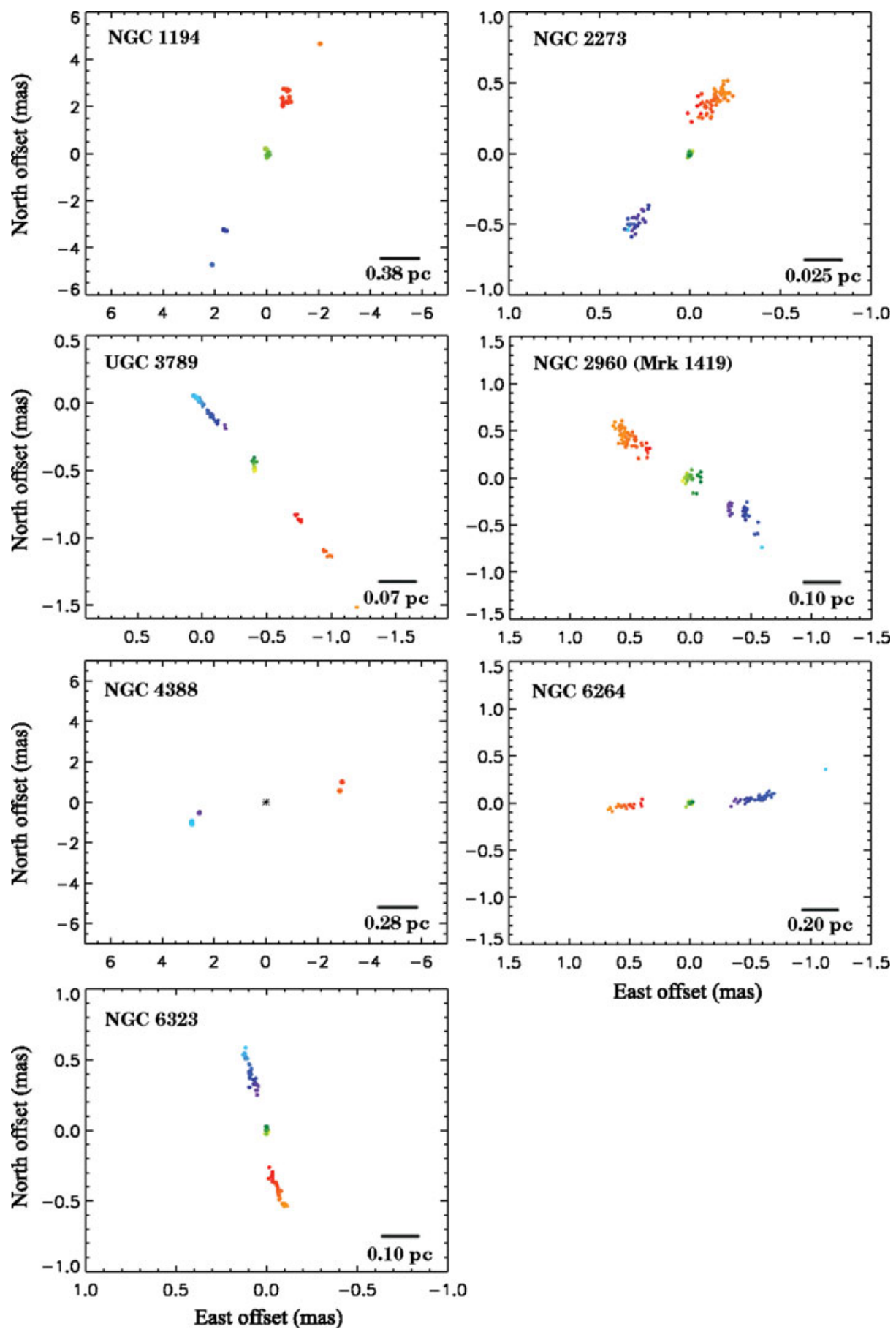

Figure 1. VLBI maps for the seven of $22 \mathrm{GHz} \mathrm{H}_{2} \mathrm{O}$ masers megamasers analyzed. The maps are color coded to indicate redshifted, blueshifted, and systemic masers, where the "systemic" masers refer to the maser components having recessional velocities close to the systemic velocity of the galaxy. Except NGC 4388, maser distributions are plotted relative to the average position of the systemic masers. For NGC 4388, in which the systemic masers are not detected, we plot the maser distribution relative to the dynamical center determined by fitting the high velocity features with a Keplerian rotation curve. 


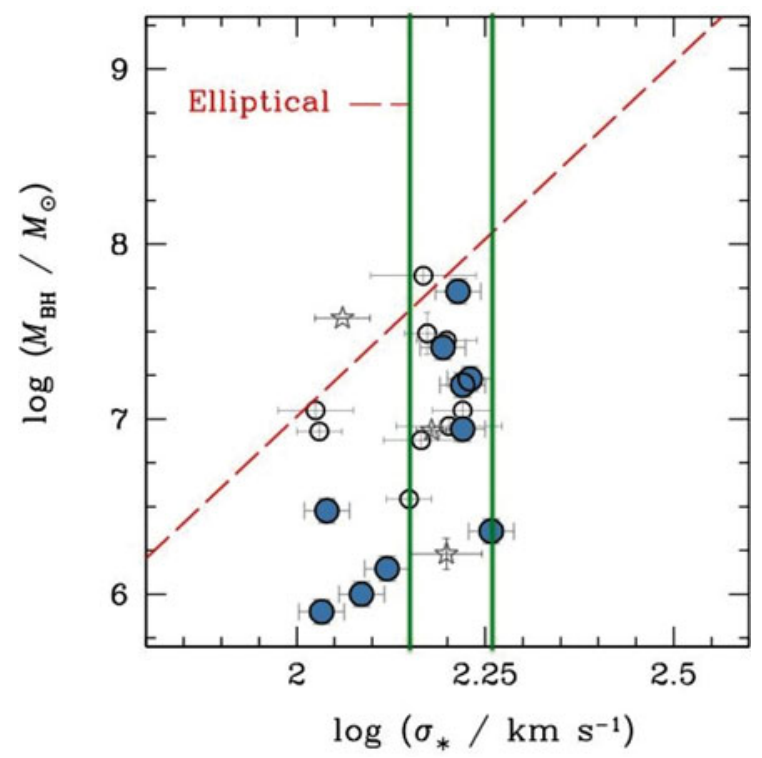

Figure 2. A plot showing the locations of $\mathrm{H}_{2} \mathrm{O}$ megamaser galaxies in the $\mathrm{M}_{B H}-\sigma_{\star}$ plane. The masers show no clear correlation. The dotted line shows the $\mathrm{M}_{B H}-\sigma_{\star}$ relation determined from early type galaxies. The galaxies bound by the two green lines in this plot emphasizes the large scatter in the BH mass for galaxies with $140 \mathrm{~km} \mathrm{~s}^{-1}<\sigma_{\star}<190 \mathrm{~km} \mathrm{~s}^{-1}$. The scatter in $\mathrm{M}_{B H}$ is nearly two orders of magnitude, too large to justify any correlation.

rotation curve, the $\mathrm{BH}$ mass is then calculated using the following equation:

$$
M_{\mathrm{BH}}=\left(\frac{\left|v_{\mathrm{K}}\right|^{2} \theta}{G}\right) D_{\mathrm{A}}=\left(\frac{\pi v_{1}^{2}}{6.48 \times 10^{8} G}\right) D_{\mathrm{A}},
$$

where $D_{A}$ is the angular diameter distance to the galaxy. In this work, we adopt the Hubble flow distance from the NASA/IPAC Extragalactic Database (NED) as $D_{\mathrm{A}}$ for the BH mass calculation.

Our new maser BH masses significantly increase the number of galaxies having dynamical $\mathrm{BH}$ masses $M_{\mathrm{BH}} \sim 10^{7} M_{\odot}$. These measurements play a particularly important role in constraining the $M-\sigma_{\star}$ relation at the low-mass end of known nuclear BH masses. With the stellar velocity dispersion $\left(\sigma_{\star}\right)$ of the galaxy bulges measured with the Dupont $2.5 \mathrm{~m}$ telescope at Las Campanas Observatory in the South and the Apache Point Observatory (APO) 3.5m telescope in the North (e.g. Greene et al. 2010), we found that $\mathrm{H}_{2} \mathrm{O}$ maser galaxies in general do not follow the $\mathrm{M}_{\mathrm{BH}}-\sigma_{\star}$ relation defined by early type galaxies (see Figure 2). From Figure 2, one can see that no correlation can be found for maser galaxies in the $M-\sigma_{\star}$ diagram, and the scatter in the BH mass for galaxies with $140 \mathrm{~km} \mathrm{~s}^{-1}<\sigma_{\star}<190 \mathrm{~km} \mathrm{~s}^{-1}$ is nearly two orders of magnitude, too large to justify any correlation.

This result is well consistent with the latest findings by Kormendy \& Ho (2013) that only early type galaxies and galaxies with classical bulges follow a tight $\mathrm{M}_{\bullet}-\sigma$ relation. Such a tight correlation may not exist in pseudo bulge galaxies.

\section{References}

Barth, A. J. Carnegie Observatories Astrophysics Series, Vol. 1: Coevolution of Black Holes and Galaxies, 2003 ed. L. C. Ho (Pasadena: Carnegie Observatories) 
Braatz, J., Condon, J., Reid, M., Henkel, C., Lo, K. Y., Kuo, C. Y., Impellizzeri, C., \& Hao, L. The Megamaser Cosmology Project Large Proposal, submitted to NRAO June 1, 2009

Braatz, J. A., Reid, M. J., Humphreys, E. M. L., Henkel, C., Condon, J. J., \& Lo, K. Y. 2010, ApJ, 718, 657

Ferrarese, L. \& Merritt, D. 2000, ApJ, 539, 9

Gebhardt, K. et al. 2000, ApJ, 539, 13

Greene, J. E., Peng, C. Y., Kim M., Kuo, C.-Y., Braatz J. A.,Impellizzeri, C. M. V., Condon, J.,Lo, F., \& Henkel C. 2010, ApJ, 721, 26

Gültekin, K. et al. 2009, ApJ, 698, 198

ormendy, J. \& Ho, L. C. 2013, ARA\&A, 51, 511

Kuo, C. Y., Braatz, J. A., Condon, J. J., Impellizzeri, C. M. V., Lo, K. Y., Zaw, I., Schenker, M., Henkel, C., Reid, M. J., \& Greene, J. E. 2011, ApJ, 727, 20

Lo, K. Y. 2005 ARA $\& A 43625$

Reid, M. J., Braatz, J. A., Condon, J. J., Greenhill, L. J., Henkel, C., \& Lo, K. Y. 2009, ApJ, 695,287 